\title{
Incorporation of Fluorescent Dyes in Electrospun Chitosan/Poly(ethylene oxide)
}

\author{
Cristiane Guellis, ${ }^{a}$ Ariane R. S. Rossin, ${ }^{b}$ Guilherme G. Bessegato, ${ }^{a}$ \\ Douglas C. Dragunski, ${ }^{\oplus a}$ Reinaldo A. Bariccattia ${ }^{a}$ and Cleber A. Lindino ${ }^{\circledR} * a$ \\ ${ }^{a}$ Grupo Interdisciplinar de Pesquisa em Fotoquímica e Eletroquímica Ambiental, \\ Departamento de Química, Universidade Estadual do Oeste do Paraná (Unioeste), \\ 85903-000 Toledo-PR, Brazil \\ ${ }^{b}$ Departamento de Química, Universidade Estadual de Maringá (UEM), \\ 87020-900 Maringá-PR, Brazil
}

\begin{abstract}
Polymeric films have been increasingly investigated due to the ease of miniaturization and integration in several sensor devices. Films obtained from the electrospinning technique have a controlled diameter and homogeneity, and substances can be incorporated into the polymeric network. Electrospinning fiber of chitosan (Ch) and poly(ethylene oxide) (PEO) was obtained from solutions prepared at different concentrations in acetic acid, and varying the distance and the voltage applied. The obtained films were characterized by scanning electron microscopy (SEM), Fourier transform infrared spectroscopy (FTIR), differential scanning calorimetry (DSC), thermogravimetric analysis (TGA), X-ray diffraction (XRD) and UV-Visible specular reflectance spectra (UV-SRS). The best conditions for electrospinning were obtained for a $2 \% \mathrm{~m} / \mathrm{v}(\mathrm{Ch}+\mathrm{PEO})$ solution in the ratio $90: 10 \% \mathrm{~m} / \mathrm{m}$ (Ch:PEO), applied voltage of $18 \mathrm{kV}$, and $18 \mathrm{~cm}$ distance between the capillary tube and collector. Acridine orange, sodium fluorescein, and erythrosine fluorescence dyes were successfully incorporated into Ch:PEO films. The spectrofluorometric spectra of the films showed excitation and emission processes and the acridine orange film showed evidence of excimer formation by the presence of an excitation peak at $569 \mathrm{~nm}$. Ch-PEO films with the incorporation of fluorescent dyes may well be used as flexible probes or sensors in colorimetric devices in biochemical applications.
\end{abstract}

Keywords: electrospinning, biopolymers, renewable polymers, fibers, luminescence

\section{Introduction}

Electrospinning is a relatively simple and versatile method for producing micro or nanoscale diameter fibers with high surface area and high porosity films. ${ }^{1}$ Electrospinning is based on the application of an electric field between a capillary tube containing the polymer solution and the collector. ${ }^{2-4}$

The production of the fibers by electrospinning depends on several parameters such as the preparation method of the solutions, the internal diameter of the needle, the distance between the needle and the collector, flow rate and applied voltage; environmental factors such as temperature and relative humidity; and solution properties such as concentration, molar mass, viscosity, conductivity, surface tension, solvent, and dielectric constant. ${ }^{2,5-7}$

*e-mail: lindino99@gmail.com
The electrospinning of various polymers of natural or synthetic origin represents a technological advance for the area. In the case of biopolymers, chitosan $(\mathrm{Ch})$ is one of the most investigated due to its characteristics of biodegradability, biocompatibility, and non-toxicity. ${ }^{8,9}$ Chitosan is a polymer derived from the deacetylation of chitin. Chitin, in turn, is a linear polysaccharide present in crustaceans, insects, and fungi and is the second most abundant saccharide material in nature after cellulose. ${ }^{10}$ Structurally, chitin is composed predominantly of units $\mathrm{N}$-acetyl-D-glucosamine and chitosan by D-glucosamine units. ${ }^{11-13}$

The solubility of chitosan is causally related to the degree of deacetylation, the $\mathrm{pH}$, and the nature of the solvent. This biopolymer is considered insoluble in solutions with a pH greater than or equal to $6.5^{6,13,14}$ and in most conventional organic solvents. ${ }^{15}$ Although inorganic acids have very limited solubility (requiring prolonged 
stirring and heating), high deacetylation chitosan is soluble in dilute organic acid solutions. ${ }^{2,69,13}$ The acid medium acts to protonate the free amino groups of this polymer to form a cationic polyelectrolyte, allowing greater solubility than its precursor, chitin. ${ }^{6,8,9}$

In the specific case of this biopolymer, it is difficult to obtain micro and nanofibers of pure chitosan. ${ }^{1,3}$ To circumvent the difficulty of electrospinning of chitosan, many studies ${ }^{1,3,9}$ evaluating its electrospinning come associated with other polymers such as poly(ethylene oxide) (PEO). PEO is a linear synthetic ethylene oxide homopolymer represented by the formula $\left(\mathrm{OCH}_{2} \mathrm{CH}_{2}\right)_{\mathrm{n}}$. It is a thermoplastic, semi-crystalline, non-toxic polymer, soluble in water and various organic solvents and used in the pharmaceutical, food, and cosmetics industries. ${ }^{16-18}$

Although the electrospinning of solutions containing only macromolecules has been extensively researched, the search for new materials has led to a growing interest in incorporating other components into the polymer matrix. Among these components, the incorporation of fluorescent dyes in polymers obtained by electrospinning can give rise to stable films for use in the medical field and the development of sensors and probes.

Several works that incorporate dyes in electrospun fibers involve chitosan blends with polyvinyl butyral (PVB), ${ }_{17}^{17}$ polycaprolactone (PCL), ${ }^{19,20}$ and polyvinyl alcohol (PVA). ${ }^{21,22}$ These are specific studies on the application of cationic dyes for the textile industry, ${ }^{17}$ such as $\mathrm{pH}$ sensors, ${ }^{19,20}$ biosensors, ${ }^{21,22}$ and $\mathrm{Hg}^{2+}$ sensor. ${ }^{23}$ They are materials customized for the desired application, also involving fast response and easy-to-view colorimetric fibrous sensors. Chitosan electrospinning results in flexible, low-cost films that immobilize dyes without impairing their chemical properties, with low leaching.

To the best of our knowledge, no studies are dealing with electrospun films of chitosan-PEO with the incorporation of fluorescent dyes. Thus, we present the investigation of the production of Ch-PEO fibers by electrospinning with the incorporation of acridine orange (AO), erythrosine $(\mathrm{E})$ and sodium fluorescein $(\mathrm{F})$.

\section{Experimental}

\section{Preparation of working solutions}

The polymers used were chitosan (Polymar Inc., Fortaleza, CE, Brazil) with $85 \%$ deacetylation degree and molar mass of 47,976.3 $\pm 1,095.5 \mathrm{~g} \mathrm{~mol}^{-1}$, determined using AVS-350 viscometer (Schott Instruments, Malnz, Germany), ${ }^{24}$ and PEO (Sigma-Aldrich, São Paulo, SP, Brazil) with a molar mass of $65,000 \mathrm{~g} \mathrm{~mol}^{-1}$. For solubilization of the polymers, $99.8 \% \mathrm{v} / \mathrm{v}$ glacial acetic acid (Neon, Suzano, SP, Brazil) and reverse osmosis purified water (Adamo, Piracicaba, SP, Brazil) were used.

Electrospinning solutions containing chitosan and PEO were prepared at concentrations of 1,2,3,4 and $5 \% \mathrm{~m} / \mathrm{v}$, of the total mass, at $90: 10$ and $95: 5 \% \mathrm{~m} / \mathrm{v}$ (Ch:PEO) ratios in $90 \% \mathrm{v} / \mathrm{v}$ acetic acid:water. To ensure complete solubilization of the solutes and obtain homogeneous material, the solutions were kept under magnetic stirring for $24 \mathrm{~h}$ at room temperature. Then, each solution was transferred to a syringe attached to the equipment of electrospinning.

\section{Polymeric fibers manufacture}

The electrospinning device includes an infusion pump (World Precision Instruments Model SP100I Syringe Pump, Sarasota, FL, USA), a high voltage source (Bertan Model 30-R, Madison Heights, MI, USA), a static metal collector with an electrical ground, and a $5 \mathrm{~mL}$ glass syringe with a hypodermic needle (internal diameter of $0.70 \mathrm{~mm}$ ).

To obtain the fibers, initial tests were performed varying the tension at $15,18,21$, and $23 \mathrm{kV}$, and the distance from the tip of the needle to the collector at 15,18 , and $20 \mathrm{~cm}$, with the flow rate kept constant at $0.5 \mathrm{~mL} \mathrm{~h}^{-1}$, according to previous experiments. These experiments were carried out at a temperature of $22 \pm 1{ }^{\circ} \mathrm{C}$ and relative humidity of $46 \pm 5 \%$. To determine the initial diameter of the fibers formed, a microscope slide was fixed on a metallic collector for the collection of samples formed in each condition.

The uniformity and diameter of the fibers from the collected slides were evaluated employing an optical microscope (Olympus, model CX31, Nishi-Shinjuku, Shinjuku-ku, Tokyo, Japan) with a camera (Olympus, model SC30, Nishi-Shinjuku, Shinjuku-ku, Tokyo, Japan) and an image capture software (Analisis Get $\mathrm{It}^{\circledR}$, Leeds, UK). Quantikov Image Analyzer 18.5 software ${ }^{25}$ (São Paulo, SP, Brazil) was used to determine the diameter, which measured ten randomly selected diameters on each slide with a $100 \times / 1.25$ objective.

\section{Factorial design}

To evaluate the influence of electrospinning parameters in the diameter of the fibers produced, a $2^{4}$ factorial design was employed using the software Chemoface ${ }^{\circledR}$ (Lavras, MG, Brazil). ${ }^{26}$ The following factors were analyzed in two levels, lower (-) and higher (+), in quadruplicate: concentration of the solution; the proportion of PEO to chitosan; distance between the syringe tip and the collector; 
and applied voltage (Table 1). The effect of each factor and its interactions were determined following Montgomery's proposal. ${ }^{27}$

Table 1. Factors and levels used in the $2^{4}$ factorial design

\begin{tabular}{lcc}
\hline \multirow{2}{*}{ Factor } & \multicolumn{2}{c}{ Level } \\
\cline { 2 - 3 } & - & + \\
\hline Concentration $(\mathrm{m} / \mathrm{v}) / \%$ & 02 & 04 \\
PEO $(\mathrm{m} / \mathrm{m}) / \%$ & 05 & 10 \\
Distance / cm & 15 & 20 \\
Applied voltage / $\mathrm{kV}$ & 15 & 21 \\
\hline
\end{tabular}

PEO: poly(ethylene oxide).

\section{Electrospinning films}

After defining the better parameters for electrospinning, new Ch-PEO solutions were prepared at a concentration of $2 \% \mathrm{~m} / \mathrm{v}$ and ratio $90: 10 \% \mathrm{~m} / \mathrm{m}(\mathrm{Ch}: \mathrm{PEO})$ to produce polymer film with dye incorporation of acridine orange $\left(\mathrm{C}_{12} \mathrm{H}_{19} \mathrm{~N}_{3}\right.$. $\mathrm{HCl} . \mathrm{ZnCl}_{2}$, Biotec, Pinhais, PR, Brazil), erythrosine $\left(\mathrm{C}_{20} \mathrm{H}_{6} \mathrm{I}_{4} \mathrm{Na}_{2} \mathrm{O}_{5}\right.$, Vetec, São Paulo, SP, Brazil) and sodium fluorescein $\left(\mathrm{C}_{20} \mathrm{H}_{10} \mathrm{Na}_{2} \mathrm{O}_{5}\right.$, Vetec, São Paulo, SP, Brazil).

The preparation of the solutions with incorporated material was conducted by solubilizing the solid chitosan and PEO in $90 \%$ v/v acetic acid, with the subsequent addition of each dye in the solution with a concentration of $2 \%$ relative to the weight of the polymers. To ensure complete solubilization these solutions were also kept under magnetic stirring for $24 \mathrm{~h}$ at room temperature. After this period, such solutions were transferred to the syringe to the electrospinning.

\section{Characterization of Ch-PEO fibers with incorporated dyes}

The morphological analysis of the films and the determination of their fiber diameters were performed by scanning electron microscopy (SEM) using equipment from the FEI company model Quanta 250 (Hillsboro, Oregon, USA). For SEM observation, the samples were fixed on a double-sided adhesive tape carbon and coated with a thin layer of gold using a Baltec SCD 005 sputter coater metallizer (Scotia, NY, USA). The fiber diameter was determined by the Quantikov Image Analyzer 18.5 software (São Paulo, SP, Brazil), ${ }^{25}$ measuring randomly the diameter of 20 fibers chosen from the SEM images, with a magnification of $10,000 \times$.

The chemical structure of chitosan and PEO powder samples and possible structural changes of these materials in the films obtained by electrospinning were evaluated by
Fourier transform infrared spectroscopy (FTIR) spectra using potassium bromide pellets $(\mathrm{KBr})$ for powder samples and attenuated total reflection (ATR) for the films. Analyzes were performed at a temperature of $25{ }^{\circ} \mathrm{C}$, in the region from 4000 to $550 \mathrm{~cm}^{-1}$, with a resolution of $8 \mathrm{~cm}^{-1}$ and 8 scans, using PerkinElmer FTIR spectrophotometer (Waltham, Massachusetts, USA).

The thermal stability of the films obtained by electrospinning and chitosan and PEO pure powders was studied by thermogravimetric analysis (TGA) on PerkinElmer model STA 6000 (Waltham, Massachusetts, USA), in the temperature range from 30 to $900{ }^{\circ} \mathrm{C}$ with $10{ }^{\circ} \mathrm{C} \mathrm{min}{ }^{-1}$ heating flow and $50 \mathrm{~mL} \mathrm{~min}{ }^{-1}$ nitrogen gas (99.999\%, White Martins, Rio de Janeiro, RJ, Brazil). The weight of the samples inserted in the porcelain crucible was 5 to $10 \mathrm{mg}$.

Thermal properties, melting processes, and crystallization (in percentage) in the films produced were determined by differential scanning calorimetry (DSC) using Shimadzu (Kyoto, Japan) DSC-60 equipment in the temperature range from 30 to $200^{\circ} \mathrm{C}$ with cooling to $30^{\circ} \mathrm{C}$, at a flow of $10^{\circ} \mathrm{C} \mathrm{min}^{-1}$ and nitrogen flow of $50 \mathrm{~mL} \mathrm{~min}^{-1}$ (99.999\%, White Martins, Rio de Janeiro, RJ, Brazil). The weight of the samples inserted into the closed aluminum crucible was 5 to $10 \mathrm{mg}$.

To calculate the crystallinity $\left(\mathrm{X}_{\mathrm{c}}\right)$, it was used the TA-60WS software (Shimadzu, Kyoto, Japan) ${ }^{28}$ and equation $1.29,30$

$\mathrm{X}_{\mathrm{c}}(\%)=\frac{\Delta \mathrm{H}_{\mathrm{f}}-\Delta \mathrm{H}_{\mathrm{c}}}{\Delta \mathrm{H}_{\mathrm{f}}^{\circ}} \times 100$

where $\Delta \mathrm{H}_{\mathrm{f}}$ is the fusion enthalpy of the sample, $\Delta \mathrm{H}_{\mathrm{c}}$ is the crystallization enthalpy and $\Delta \mathrm{H}_{\mathrm{f}}^{\circ}$ is the theoretical fusion enthalpy of the $100 \%$ crystalline sample.

The determination of the degree of crystallinity of the chitosan and PEO powder and the possible structural alterations of these materials and dyes in the produced films was performed by X-ray diffraction (XRD) in Bruker $^{\circledR}$ model D2 Phaser equipment (Billerica, Massachusetts, USA), with $\mathrm{Cu} \mathrm{K} \alpha$ radiation of $\lambda=1.5406 \AA$, $2 \theta$ angle scan from 5 to $60^{\circ}$ and increment of $0.02^{\circ}$.

Determination of the maximum absorption wavelength of the dyes in $0.001 \% \mathrm{~m} / \mathrm{v}$ solutions in purified water was carried out in the range from 190 to $1100 \mathrm{~nm}$, with a $10 \mathrm{~mm}$ optical path quartz cuvette and $1 \mathrm{~nm}$ slit in a spectrophotometer UV-Vis Shimadzu model UV-1800 (Kyoto, Japan). Specular reflectance spectra of electrospinning films were also performed on this spectrophotometer, in the range of 190 to $1100 \mathrm{~nm}$, with a $5^{\circ}$ angle reflectance accessory. 
The fluorescence spectra of the dyes in the films were obtained from an Aminco model SPF-500C spectrophotometer (Urbana, Illinois, USA), equipped with a $300 \mathrm{~kW}$ xenon discharge lamp and photomultiplier voltage at $800 \mathrm{~V}$, with the excitation and emission slits maintained at $4 \mathrm{~nm}$.

\section{Results and Discussion}

\section{Polymeric fibers manufacture}

Before the execution of the factorial design, exploratory experiments were carried out to investigate the electrospinning conditions in which fibers are obtained. There is no formation of pure chitosan fibers at 2 and $4 \% \mathrm{~m} / \mathrm{v}$ in the electrospinning process, but only of droplets deposited in the collector, even after changes in the experimental conditions. These results corroborate the literature, $1,3,10,14,31,32$ about the difficulty of producing nanofibers of pure chitosan.

The difficulty in electrospinning of pure chitosan is mainly due to the high viscosity of its solution, which can be explained by the strong hydrogen bonds between the polymeric chains and their intertwining which restrict their mobility. ${ }^{33}$ Due to the intense electric field, such links impede the free movement of the polymer chain, leading to disruption of the jet during the electrospinning process. Also, repulsive forces between the $\mathrm{NH}_{3}$ groups of this biopolymer may prevent sufficient chain tangles from forming for continuous fiber formation during jet stretching, "whipping" and "flexing" resulting in droplet production in the collector. ${ }^{1}$ Based on the literature, ${ }^{1,3,7,8}$ the PEO was used together with chitosan as an additive to facilitate the fiber formation.

The solutions of 2,3 , and $4 \% \mathrm{~m} / \mathrm{v}$ of the polymers, in the $90: 10$ and $95: 5 \% \mathrm{~m} / \mathrm{m}$ ratio (Ch:PEO) allowed the formation of fibers without the presence of beads. The addition of the PEO facilitated the electrospinning of this biopolymer to reduce the viscosity and electric conductivity of the chitosan solution due to hydrogen bonds formed between the amino groups of the polymer and PEO ether groups, which helps in obtaining a more stable jet..$^{1,93}$

\section{Factorial design}

The Pareto chart (Figure 1) shows the effect values and which factors were statistically significant $(p$-value $=0.05)$ in the fiber diameter, from the factorial design $2^{4}$.

The most statistically significant factors were the mass of chitosan and PEO dissolved in the acetic acid solution, the proportion (in percentage) of PEO to chitosan, and

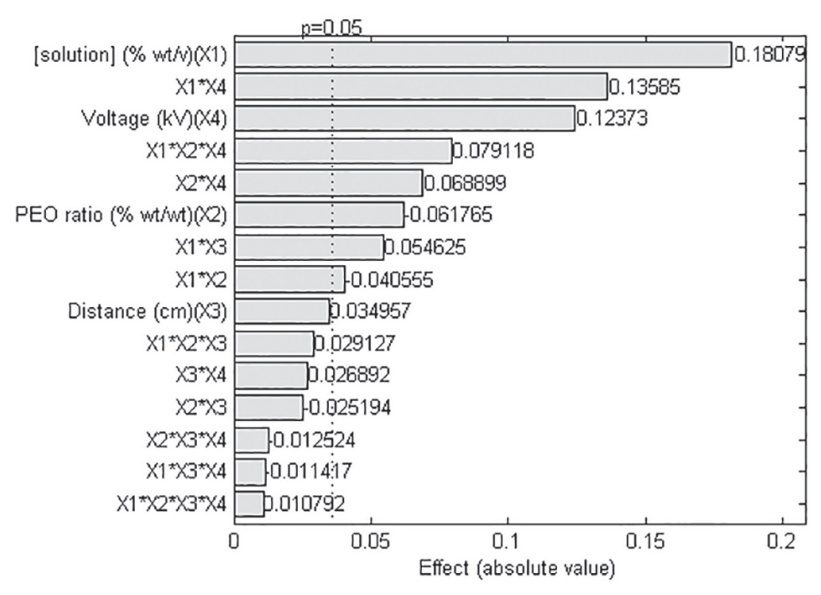

Figure 1. Pareto chart showing the effects of solution concentration (X1), $\mathrm{Ch}$ :PEO ratio (X2), the distance between the needle and the collector (X3) and applied voltage (X4) on the average diameter of Ch-PEO fibers.

the applied voltage. The distance between the needle and the collector does not influence the diameter of the fibers formed. Considering the calculation of the influence of each factor according to Montgomery, ${ }^{27}$ increasing the concentration of the solution may produce thicker fibers, as chitosan is the predominant component and the solutions with the highest concentrations of this polymer are more viscous. ${ }^{7}$ The increase in the proportion of PEO results in reduced diameter of the fibers since the addition of PEO reduces the viscosity of the chitosan solution. ${ }^{9,33}$

In the electrospinning process, the trend is that the increasing voltage decreases the diameter of the fibers. ${ }^{4}$ According to Zhang et al. ${ }^{34}$ increasing the intensity of the electric field increases the repulsive electrostatic force in the jet, which favors the formation of smaller fibers in diameter. However, it was observed that the increased voltage resulted in larger fibers. A possible explanation for the results is due to the interaction between applied voltage and percentage of the mass of the polymers in the solution, with the solution at the tip of the needle being removed more quickly as the jet is ejected from the Taylor cone, increasing the diameter of the fiber. ${ }^{34}$

Since the objective is to make fibers with a smaller diameter to obtain the largest possible surface area and allowing more effective incorporation of dyes, it was adopted the electrospinning parameters that produced the lowest standard deviation of fiber diameters which indicates a greater homogeneity. In this case, we used the $2 \% \mathrm{~m} / \mathrm{v}$ solution at a ratio of $90: 10 \% \mathrm{~m} / \mathrm{m}$ (Ch:PEO), an applied voltage of $18 \mathrm{kV}$, and a distance of $18 \mathrm{~cm}$. Figure 2 shows the SEM image of the obtained fibers. The SEM image shows that the electrospinning under these conditions did not produce beads. The average fiber diameter was $0.2855 \pm 0.0508 \mu \mathrm{m}(\mathrm{n}=10)$, corroborating the results obtained by other authors. ${ }^{3,9,10,12,34}$ 


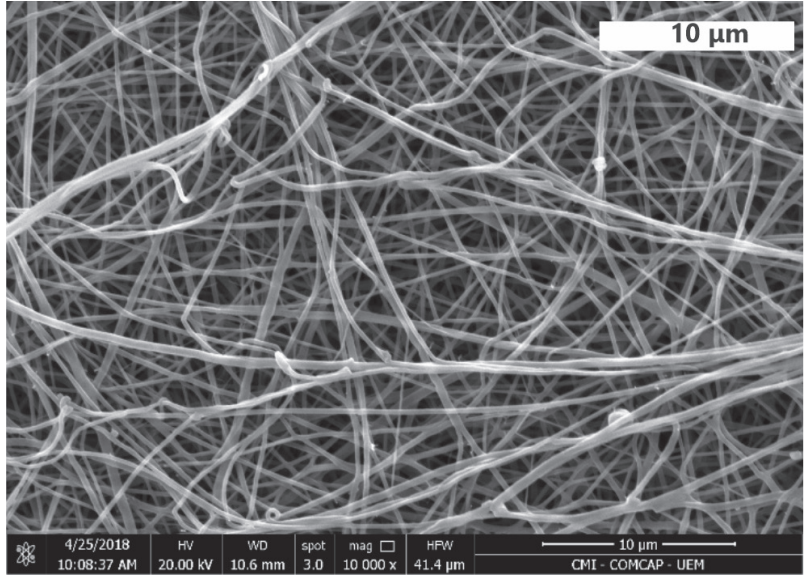

Figure 2. SEM micrograph $(10,000 \times)$ of Ch-PEO electrospun fibers produced from the $2 \% \mathrm{~m} / \mathrm{v}$ concentration solution and $90: 10 \% \mathrm{~m} / \mathrm{m}$ (Ch:PEO) at a distance of $18 \mathrm{~cm}$ and an applied voltage of $18 \mathrm{kV}$.

\section{Characterization of Ch-PEO with incorporated dyes}

The incorporation of the fluorescent dyes did not produce changes in the morphology of the fibers (Figure 3). The fibers were uniform and without the presence of beads. The average diameter obtained was $0.2472 \pm 0.0436$, $0.2492 \pm 0.0355$ and $0.2558 \pm 0.0424 \mu \mathrm{m}$ for the acridine orange (Ch-PEO/AO) erythrosine (Ch-PEO/E) and sodiumfluorescein $(\mathrm{Ch}-\mathrm{PEO} / \mathrm{F})$, respectively. By $t$-Student's test, there is no statistically significant difference between the average diameters of fibers produced without or with dyes.

Dye absorption spectra in $0.001 \% \mathrm{~m} / \mathrm{v}$ aqueous solution (Figure 4) show that acridine orange has a maximum absorption wavelength of $470 \mathrm{~nm}$ and a shoulder at $491 \mathrm{~nm},{ }^{35-37}$ erythrosine at $526 \mathrm{~nm},{ }^{38,39}$ and sodium fluorescein
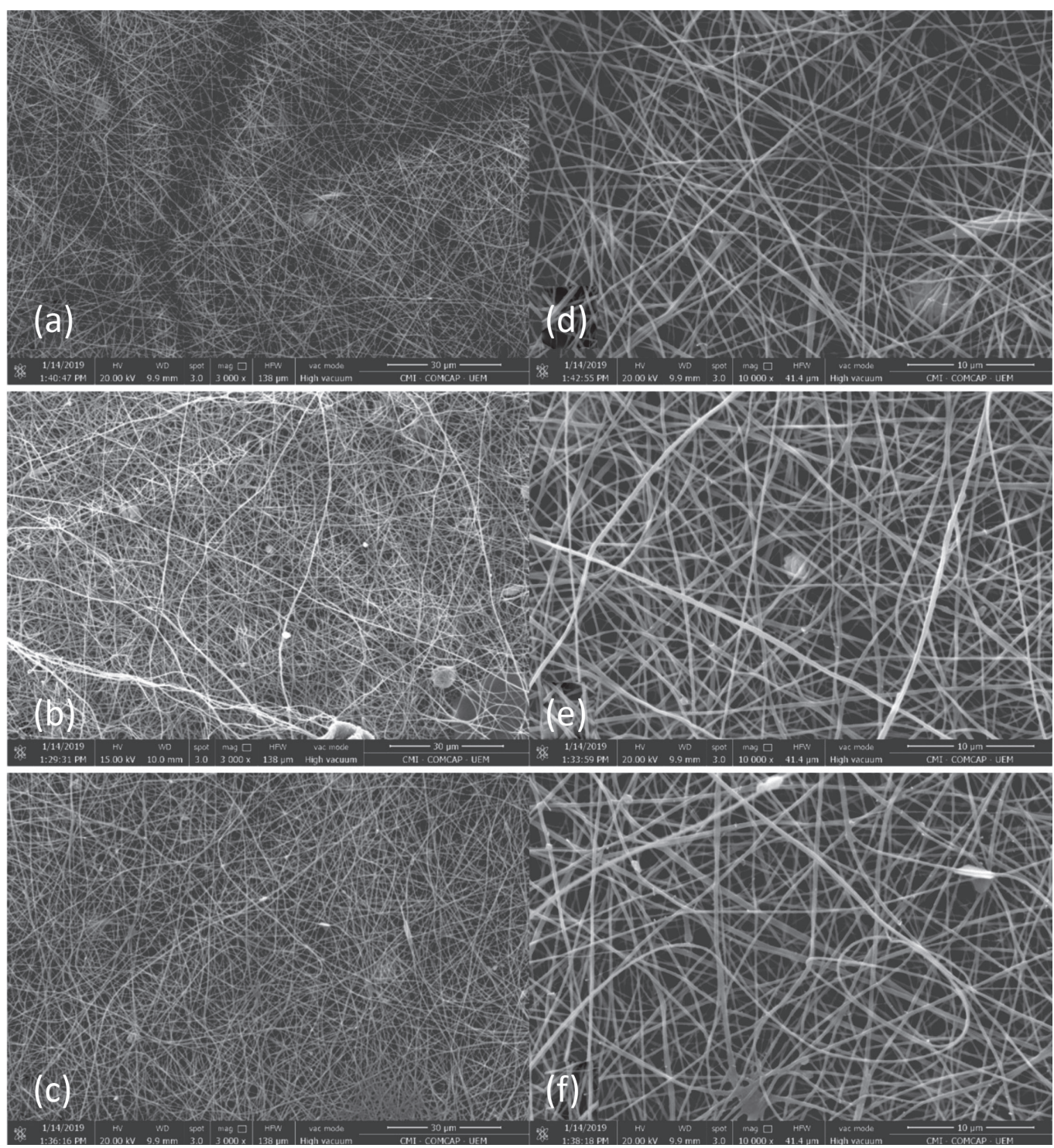

Figure 3. Scanning electron microscopy images of the Ch-PEO electrospun films with $2 \%$ incorporation of the orange dyes: acridine (a, d), erythrosine (b, e), and sodium fluorescein (c, f), to the mass of the polymers. Conditions: electrospinning of chitosan and PEO solutions at $2 \% \mathrm{~m} / \mathrm{v}$, in the ratio $90: 10 \% \mathrm{~m} / \mathrm{m}$, under an applied voltage of $18 \mathrm{kV}$ and a distance of $18 \mathrm{~cm}$ between needle and collector. Magnifications of 3,000×, $30 \mu \mathrm{m}$ (left) and 10,000×, 10 $\mu \mathrm{m}$ (right). 
at 454 and $478 \mathrm{~nm} \cdot{ }^{40}$ Concerning acridine orange, the increase in the concentration of this dye in solution changed the absorption band profile, which shows an increase in the intensity of $470 \mathrm{~nm}$, attributed to the dimer. ${ }^{35,36}$

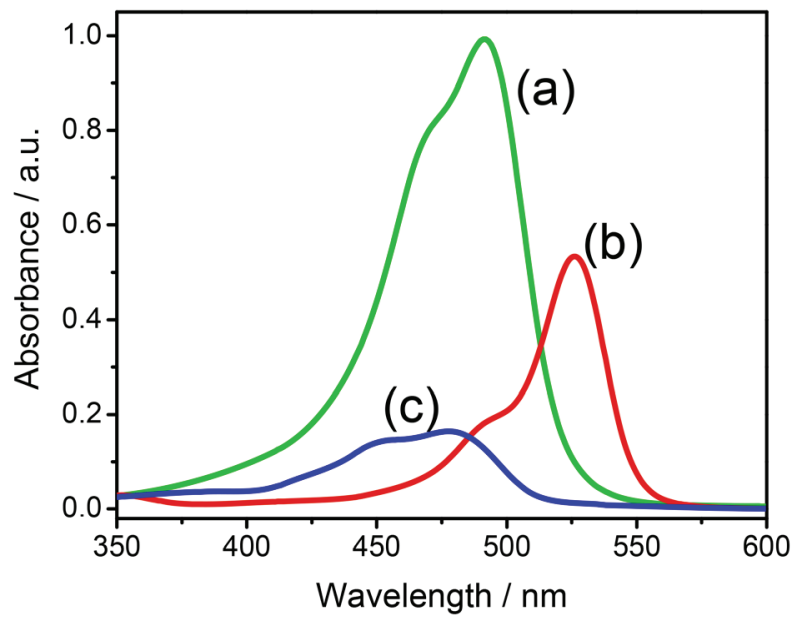

Figure 4. Absorption spectra of (a) acridine orange, (b) erythrosine, and (c) sodium fluorescein dyes at $0.001 \% \mathrm{~m} / \mathrm{v}$ in aqueous solution.

The incorporation of dyes in the electrospinning films can be verified by the UV-Vis reflectance spectra, Figure 5a. The maximum reflectance values of 491 and 470 (acridine orange), 541 (erythrosine), and $505 \mathrm{~nm}$ (sodium fluorescein) are close to the maximum absorption of the dyes in solution (Table 2). In the case of acridine orange, changes in the reflectance spectrum profile may indicate the presence of dye aggregates. ${ }^{36,37}$

Figure 6 shows the fluorescence spectra. Fluorescence spectra of sodium fluorescein and erythrosine dyes (Figures $6 \mathrm{c}$ and $6 \mathrm{~d}$ ) are very similar to those obtained in solution. Stokes displacement was $32 \mathrm{~nm}$ for erythrosine and $79 \mathrm{~nm}$ for sodium fluorescein. ${ }^{40,41}$

The acridine orange dye in Ch-PEO films showed very different emission spectra (Figure 6a). ${ }^{37}$ In this case, the

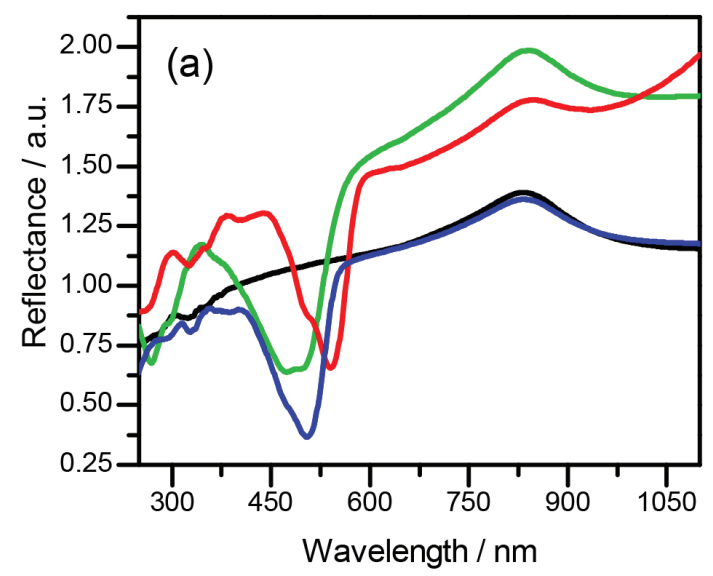

Table 2. Maximum absorption wavelength values of the dyes in $0.001 \% \mathrm{~m} / \mathrm{v}$ aqueous solution and reflectance wavelength in the electrospun films

\begin{tabular}{lccc}
\hline Dye & $\lambda$ solution $/ \mathrm{nm}$ & $\lambda$ literature $/ \mathrm{nm}$ & $\lambda(\log 1 / \mathrm{R}) / \mathrm{nm}$ \\
\hline Acridine orange & 470 and 491 & 467 and $490^{35-37}$ & 482 \\
Erythrosine & 526 & $525^{38,39}$ & 539 \\
$\begin{array}{l}\text { Sodium } \\
\text { fluorescein }\end{array}$ & 454 & $457^{40}$ & 502 \\
\hline
\end{tabular}

$\lambda$ : wavelength; R: reflectance.

large-format emission band is assigned to monoprotonated specie ( $\mathrm{p} K_{\mathrm{a}}$ of $\mathrm{AO}$ is 9.8 ), due to the dissolution of the dye in $90 \% \mathrm{~m} / \mathrm{v}$ acetic acid during the preparation of the electrospun films. The presence of the emission acute peak at $604 \mathrm{~nm}$, is unusual. As no bands were observed at this wavelength in the reflectance spectrum of the film, this peak may be indicative of excimer (excited dimer) formation. ${ }^{36}$

Excimers are excited-singlet state dimers ("excited + dimer" = excimer) ${ }^{36,42}$ According to Hubenko et al. ${ }^{36}$ and Sarkar et $a l .{ }^{43}$ there are two types of excimers: dynamics and statics. The dynamic excimer is formed by the collision between a fluorophore in the ground state and a fluorophore in the excited state, during the lifetime of the excited species. ${ }^{36,42,43}$ In contrast, the static excimer is formed from a pre-arranged dimer in the ground state. In this situation, the monomeric units of the dimer must be arranged in such a way that the distance between them is short enough to form the excimer by photo-excitation. According to Hubenko et al., ${ }^{36}$ this occurs due to fluorophores concentrated on solid surfaces and porous matrices, that is, in media with restricted geometry. ${ }^{44}$ This phenomenon could indicate the interaction of the fluorescent dye in the electrospun fibers, making the molecule more rigid, reducing vibrations, and promoting the possibility of occurrence of excimers. Excimer state is not observed in the absorption spectrum. ${ }^{45}$

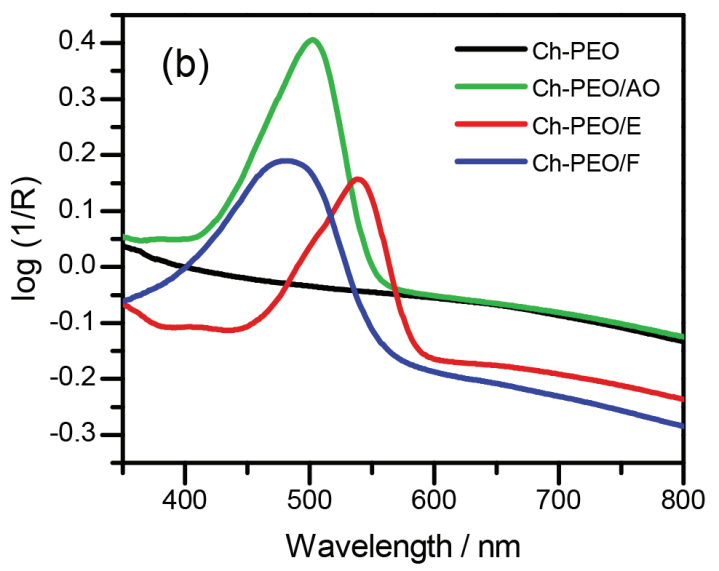

Figure 5. (a) Reflectance spectra and (b) absorbance (log (1/R)) of electrospun dye-impregnated films in visible spectra: (一) Ch-PEO without dyes, $(-)$ Ch-PEO/AO, (-) Ch-PEO/E and (-) Ch-PEO/F. 


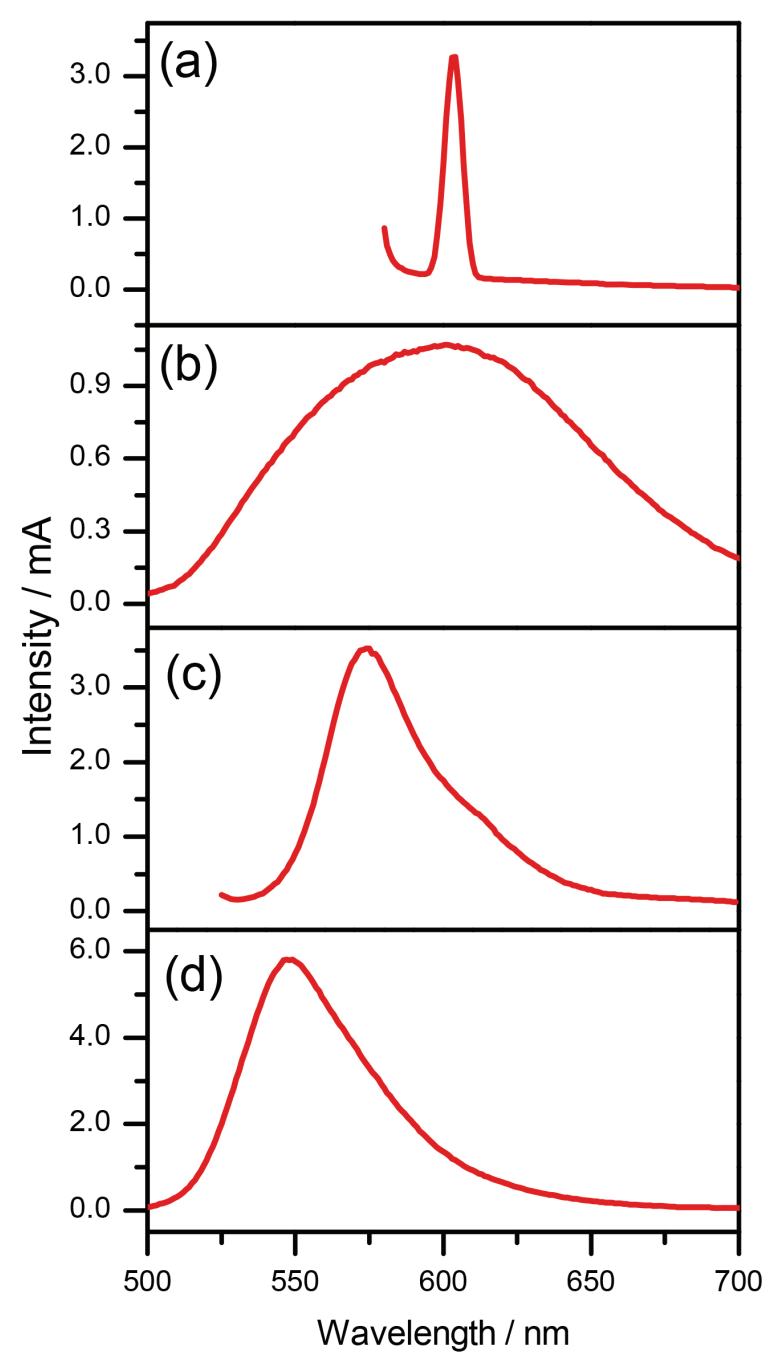

Figure 6. Fluorescence spectra (emission) of the (a) Ch-PEO/AO $\left(\lambda_{\text {exc. }}=570 \mathrm{~nm}\right)$; (b) Ch-PEO/AO $\left(\lambda_{\text {exc. }}=473 \mathrm{~nm}\right)$; (c) Ch-PEO/E and (d) $\mathrm{Ch}-\mathrm{PEO} / \mathrm{F}$.

In the FTIR characterization, the main bands of the $\mathrm{Ch}$ and PEO powder samples, electrospinning films without fluorescent dyes can be seen in Figure 7A. Table 3 shows the main bands for $\mathrm{Ch}$ and $\mathrm{PEO}$ powder samples.

In the $\mathrm{Ch}-\mathrm{PEO}$, the protonation of the $\mathrm{Ch}$ amine group gives rise to bands of 1551 and $1407 \mathrm{~cm}^{-1} .50,51$ Furthermore, change in intensity of the band at $1060 \mathrm{~cm}^{-1}$ to $\mathrm{Ch}$ and $\mathrm{Ch}-\mathrm{PEO}$ and 1071 and $1064 \mathrm{~cm}^{-1}$ to Ch-PEO, respectively, may be indicative of the displacement of the $\mathrm{C}=\mathrm{O}$ band to smaller wavenumbers, which corroborates the results of Pakravan et al., ${ }^{1}$ and indicates hydrogen bond interactions between the $\mathrm{Ch}$ amino group and the PEO ether group. According to Aluigi et al.,$^{52}$ the stretching of the polymer in the electrospinning promotes the orientation of the polymeric chains that leads to this change in conformation.

The inhibition of the bands at 1551 and $1407 \mathrm{~cm}^{-1}$ (Figure 7B), attributed to the group $\mathrm{NH}_{3}{ }^{+}$is the effect of the incorporation of dyes in the films obtained by
Table 3. Main bands in the infrared region of chitosan $(\mathrm{Ch})$ and poly(ethylene oxide) (PEO) powder samples

\begin{tabular}{|c|c|c|}
\hline $\begin{array}{l}\text { Wavenumber / } \\
\mathrm{cm}^{-1}\end{array}$ & Assignment & Reference \\
\hline \multicolumn{3}{|c|}{ Chitosan } \\
\hline 3449 & $\mathrm{OH}$ and $\mathrm{NH}_{2}$ axial deformation & 33,46 \\
\hline 2925 and 2876 & $\begin{array}{c}\mathrm{C}-\mathrm{H} \text { antisymmetric and symmetric } \\
\text { stretching }\end{array}$ & 47,48 \\
\hline 1649 & $\mathrm{C}=\mathrm{O}$ axial deformation of amide & $47-49$ \\
\hline 1557 & $\mathrm{~N}-\mathrm{H}$ angular deformation & 48 \\
\hline 1422 & $\mathrm{CH}_{2}$ angular deformation & 33,48 \\
\hline 1383 & $\mathrm{CH}_{3}$ symmetric deformation & 46,48 \\
\hline 1152 & $\mathrm{C}-\mathrm{O}-\mathrm{C}$ axial deformation & 47,48 \\
\hline 1092 & $\mathrm{C}-\mathrm{O}$ axial deformation & 46 \\
\hline \multicolumn{3}{|c|}{ Poly(ethylene oxide) } \\
\hline 3488 & O-H stretching & 46 \\
\hline 2886 & $\mathrm{CH}_{2}$ axial deformation & 33 \\
\hline 1466 and 1348 & $\mathrm{CH}_{2}$ angular deformation & 33 \\
\hline 1100 & $\mathrm{C}-\mathrm{O}-\mathrm{C}$ axial stretching & 9 \\
\hline
\end{tabular}

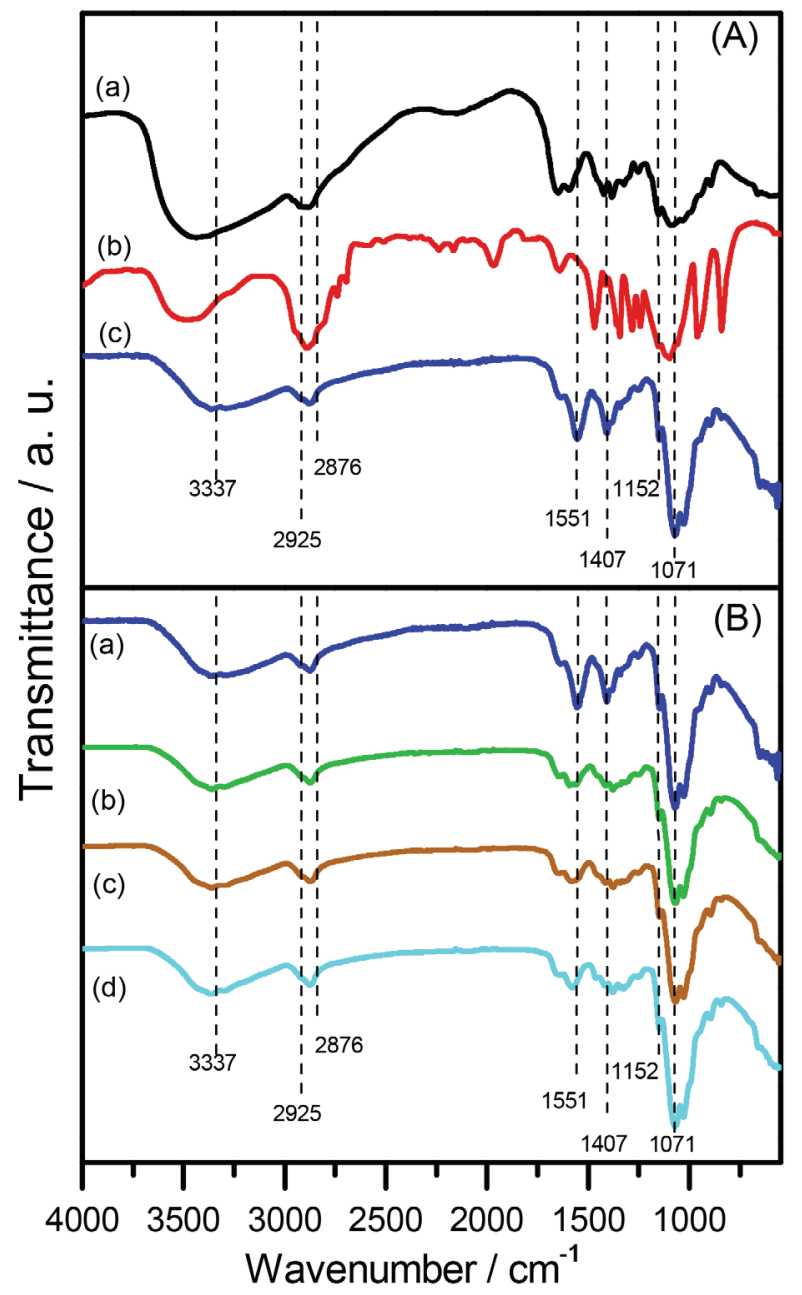

Figure 7. (A) FTIR spectra of (a) Ch and (b) PEO powder samples; and of (c) Ch-PEO electrospun film. (B) FTIR spectra of electrospun films: (a) Ch-PEO, (b) Ch-PEO/AO, (c) Ch-PEO/E, and (d) Ch-PEO/F. 
electrospinning. This indicates that the greatest interaction of the dyes with the polymers occurred with the Ch amine group. As the ratio of the polymers in the solution is $90: 10 \% \mathrm{~m} / \mathrm{m}(\mathrm{Ch}: \mathrm{PEO})$ there is a probability of several free groups in $\mathrm{Ch}$ that could interact with the dyes.

Thermogravimetric analysis (Figure 8a) showed that the Ch curve has two distinct thermal events of mass loss. The first event occurred between 30 and $100{ }^{\circ} \mathrm{C}$, and it was attributed to the water loss $(11 \%)$ present in the biopolymer. The second event, starting at 289 to $375^{\circ} \mathrm{C}$, showed maximum degradation at $305^{\circ} \mathrm{C}$ with $43 \%$ loss of material and is attributed to $\mathrm{Ch}$ degradation, ${ }^{3}$ which has no total weight loss and leaves residual material. ${ }^{53}$

In the case of PEO, the thermogravimetric curve shows only one weight-loss event, corresponding to its degradation, which starts at $373{ }^{\circ} \mathrm{C}$, has a maximum value at $399^{\circ} \mathrm{C}$ and continues until $425^{\circ} \mathrm{C}$, with intensity decrease until total weight loss at $569^{\circ} \mathrm{C} .{ }^{3}$

Three distinct and characteristics of thermal loss events are present for Ch-PEO film. The first event is the loss of water that corresponds to $15 \%$ of the weight. The second event is the degradation of $\mathrm{Ch}$, which starts at $264{ }^{\circ} \mathrm{C}$ with a maximum at $283{ }^{\circ} \mathrm{C}$, and the third event corresponds to the degradation of $\mathrm{PEO}$ at $312{ }^{\circ} \mathrm{C}$ with a maximum at $389^{\circ} \mathrm{C}$. According to Bonardd et al.,$^{54}$ the difference in the onset of events of electrospun films compared to powdered polymers is due to the intermolecular interactions between the components of the mixture, which, in turn, modify the thermal stability of the polymers corroborating with the results obtained by FTIR analysis. ${ }^{49}$

TGA also showed that the electrospinning production of the films changed the degradation temperature of the polymers. In the case of Ch-PEO, the amount of water and solvent is lower and the thermal stability of $\mathrm{Ch}$ decreased while that of PEO increased. In the dye-incorporated Ch-PEO films, there was a decrease in Ch degradation temperature and an improvement in PEO thermal stability when compared to $\mathrm{Ch}-\mathrm{PEO}$, indicating polymer interactions with the dyes. In the case of Ch-PEO/E film specifically, possible interactions result from the nitrogen in amine groups in chitosan and iodine atoms in erythrosine. Carbonbound halogens can be involved in attractive interactions with a nucleophile with at least one pair of free electrons (such as $\mathrm{N}$ ). The strength of the interaction increases on moving from chlorine to bromine to iodine. ${ }^{55,56}$

The data presented in Figure $8 \mathrm{~b}$ corroborate with the data observed in Figure 8a and also indicates changes in the degradation profile of the polymers, depending on how to obtain the blend or the incorporation of dyes.

Differential scanning calorimetry (DSC) results are shown in Figure 9. The DSC curve for Ch powder exhibits

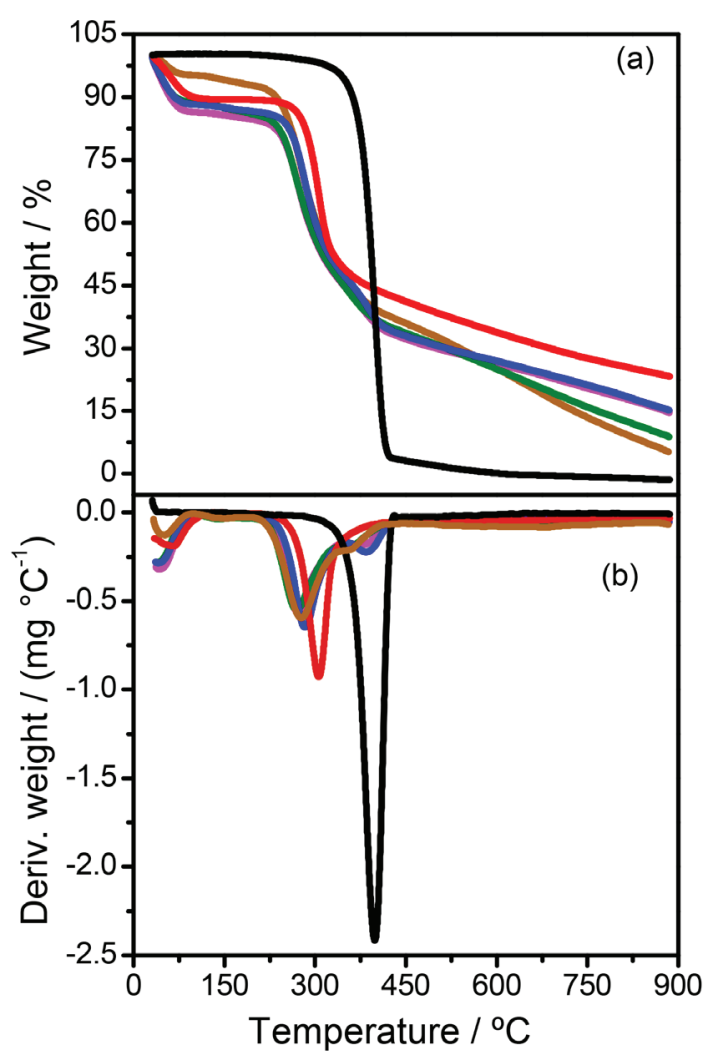

Figure 8. (a) Thermogravimetric curves and (b) derived from the thermogravimetric curves of the obtained polymers: (-) PEO powder; (-) Ch powder; (-) Ch-PEO electrospun film; (-) Ch-PEO/AO electrospun film; (-) Ch-PEO/E electrospun film; (-) Ch-PEO/F electrospun film.

only one endothermic peak at approximately $100{ }^{\circ} \mathrm{C}$, attributed to water loss, ${ }^{3,57}$ which is confirmed by the weight loss detected in the first thermal event of the TGA and DTG curves discussed earlier (Figures 8a and 8b). According to the literature, ${ }^{54,58}$ chitosan (as well as other polysaccharides) is degraded before its melting temperature is reached due to hydrogen bonding when subjected to elevated temperatures. In the case of PEO powder (Figure 9a), the DSC curve shows an acute endothermic peak around $75{ }^{\circ} \mathrm{C}$, which corresponds to the melting of this polymer, and another exothermic peak around $50{ }^{\circ} \mathrm{C}$, which refers to its crystallization. ${ }^{3,59}$

For Ch-PEO, the presence of the endothermic peak around $50^{\circ} \mathrm{C}$ corresponds to the fusion of PEO, that in the presence of $\mathrm{Ch}$ occurs at lower temperatures, due to the interaction of polymers ${ }^{3,55}$ confirmed by infrared analysis. In turn, the endothermic peak present at approximately $80{ }^{\circ} \mathrm{C}$ refers to the loss of water. ${ }^{3,49}$ Thus, the exothermic peak in the cooling curve around $43{ }^{\circ} \mathrm{C}$ corresponds to PEO crystallization, which also occurs at a lower temperature due to the influence of $\mathrm{Ch}^{3}{ }^{3}$

The incorporation of the dyes did lead to changes in blend melting and crystallization temperatures compared to 
PEO (Table 4) and consequently changes the heat involved in each process with the decrease of the crystallinity of the films, indicating an interaction of the materials with the PEO, which corroborates the data obtained in the FTIR and TGA analyses.

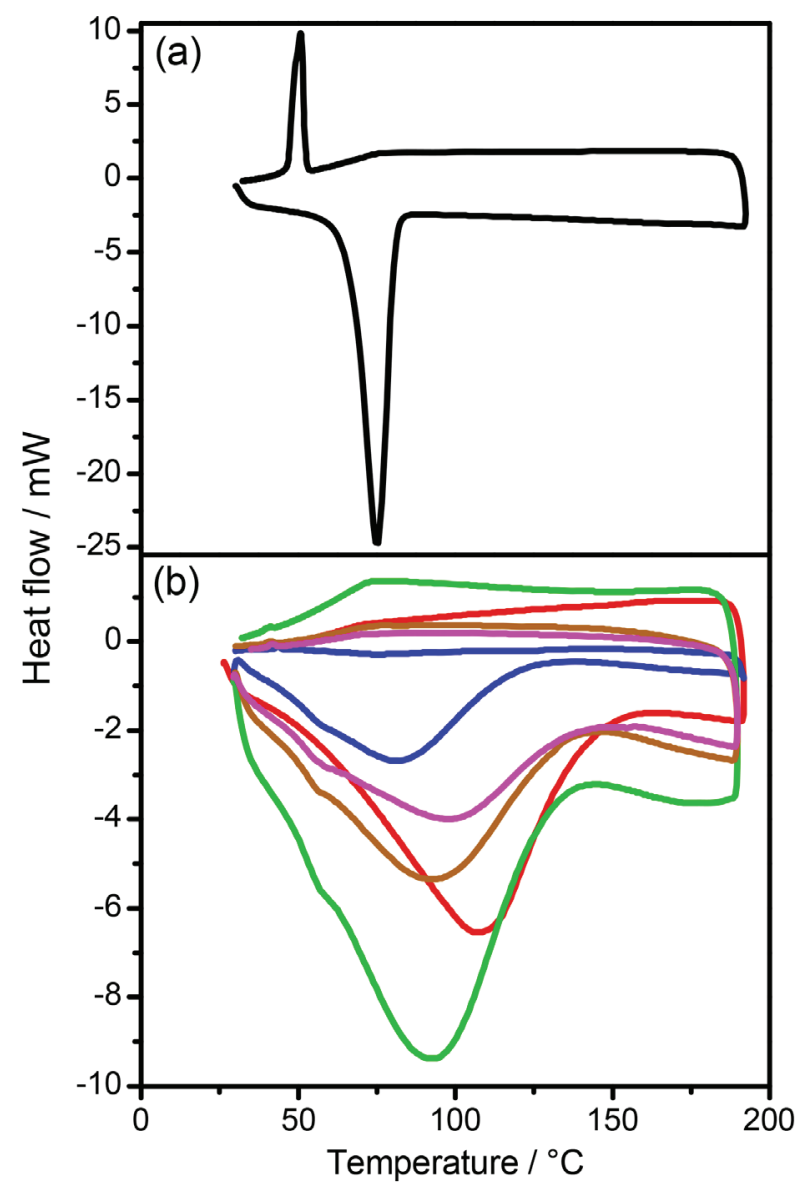

Figure 9. Differential scanning calorimetry curves obtained for the materials: (a) PEO powder; (b) (-) Ch powder; (-) Ch-PEO electrospun film; (-) Ch-PEO/AO electrospun film; (-) Ch-PEO/E electrospun film; (一) Ch-PEO/F electrospun film.

For the calculation of the crystallinity percentage of the samples, which in this case refers to the PEO, the values of the heat involved in the fusion and crystallization processes are presented in Table 4. The value of theoretical fusion enthalpy of $100 \%$ crystalline PEO is $213 \mathrm{~J} \mathrm{~g}^{-1} .^{16}$

The remarkable decrease in crystallinity for the Ch-PEO blends with incorporated dyes to pure PEO is confirmed by the significant decrease in the peaks observed in X-ray diffractometry (Figure 10). X-ray diffraction (Figure 10) showed that PEO is a high crystallinity polymer and Ch a semi-crystalline biopolymer. ${ }^{9,57}$ In the case of the Ch-PEO diffractogram, there is only a low-intensity peak around $29^{\circ}$ confirming the amorphous behavior of the film, which corroborates the DSC analysis. For Kuntzler et al. ${ }^{57}$ this result may be related to the orientation of the PEO
Table 4. Temperatures and enthalpies of melting and crystallization and percent crystallinity of samples

\begin{tabular}{lccccc}
\hline Sample & $\mathrm{Mt} /{ }^{\circ} \mathrm{C}$ & $\begin{array}{c}\Delta \mathrm{H}_{\mathrm{f}} / \\
\left(\mathrm{J} \mathrm{g}^{-1}\right)\end{array}$ & $\mathrm{C}_{\mathrm{t}} /{ }^{\circ} \mathrm{C}$ & $\begin{array}{c}\Delta \mathrm{H}_{\mathrm{c}} / \\
\left(\mathrm{J} \mathrm{g}^{-1}\right)\end{array}$ & $\mathrm{X}_{\mathrm{c}} / \%$ \\
\hline PEO powder & 75.49 & 158.92 & 50.71 & -108.77 & 23.54 \\
Ch-PEO/AO & 51.78 & 2.75 & 43.72 & -0.67 & 0.97 \\
Ch-PEO/E & 50.99 & 2.58 & 41.27 & -0.75 & 0.85 \\
Ch-PEO/F & 51.70 & 3.21 & 41.72 & -1.09 & 0.99 \\
\hline
\end{tabular}

PEO: poly(ethylene oxide); Mt: melting temperature; $\Delta \mathrm{H}_{\mathrm{f}}$ : fusion enthalpy; $\mathrm{C}_{\mathrm{t}}$ : crystallization temperature; $\Delta \mathrm{H}_{\mathrm{c}}$ : crystallization enthalpy; $\mathrm{X}_{\mathrm{c}}$ : percentage of crystallinity; Ch: chitosan; $\mathrm{AO}$ : acridine orange; E: erythrosine; F: sodium fluorescein.

crystals due to the application of high voltage during the electrospinning process. Moreover, the low crystallinity of PEO in films can be justified by the interaction of this polymer with $\mathrm{Ch}$ via hydrogen bonding. ${ }^{60}$

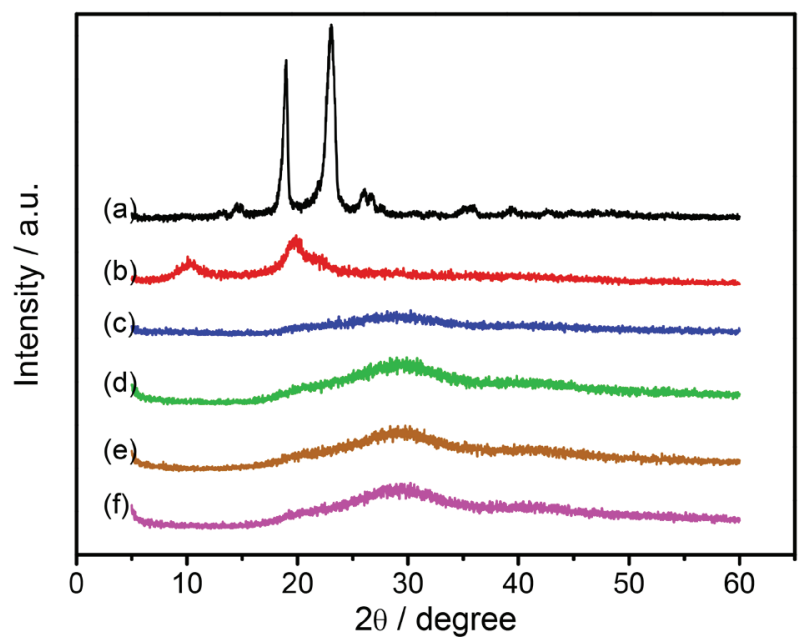

Figure 10. X-ray diffractograms of the samples: (a) PEO powder, (b) $\mathrm{Ch}$ powder; (c) Ch-PEO electrospun film, (d) Ch-PEO/AO, (e) Ch-PEO/E and (f) $\mathrm{Ch}-\mathrm{PEO} / \mathrm{F}$ (electrospun films).

The incorporation of these fluorescent dyes in electrospun biopolymeric fibers is an important achievement towards the production of flexible films with potential use in biomedical applications.

Preliminary leaching tests of the dyes, in purified water, over time, from electrospuns films with an area of $4 \mathrm{~cm}^{2}$ and absorption measures in the visible region, indicated the loss of $8.39 \times 10^{-10} \mathrm{~mol} \mathrm{~h}^{-1} \mathrm{~cm}^{-2}$ for erythrosine and $7.02 \times 10^{-10} \mathrm{~mol} \mathrm{~h}^{-1} \mathrm{~cm}^{-2}$ for sodium fluorescein. The data for acridine orange were not conclusive. This data is relevant because it shows that the dyes leached in small quantities, not interfering in biomedical applications.

For instance, erythrosine could be used as a membrane with antifungal and antibacterial activity, ${ }^{61,62}$ and the fluorescein-incorporated fiber as sensors. ${ }^{63}$ Acridine orange was used as a probe for deoxyribonucleic acid 
(DNA) and ribonucleic acid (RNA) discrimination ${ }^{37,64}$ and has applications in photodynamic therapy, detection of gynecological cancer, and rapid diagnosis of malaria. ${ }^{65,66}$ Acridine orange fibers could be applied directly to skin cancer regions in photodynamic therapies. The dyes incorporated in the electrospun films are less susceptible to fluorescence quenching caused by changes in temperature, oxygen dissolved in the liquid medium, or due to the transfer of non-radiant energy to the solvent.

\section{Conclusions}

The incorporation of the acridine orange, erythrosine, and sodium fluorescein dyes in biodegradable, biocompatible, and non-toxic electrospun films of chitosan-PEO was obtained successfully. The luminescent emission of each of the dyes was obtained in the solid-state, allowing to stabilize the dyes, reducing interactions with solvents that cause quenching of the emitted radiation, opening new fields of study for the development of flexible sensors and fluorescent probes, in medical diagnostics, food or pharmaceuticals applications.

\section{Acknowledgments}

The authors are grateful for the financial support provided by the Brazilian funding agencies. This study was financed in part by the Coordenação de Aperfeiçoamento de Pessoal de Nível Superior Brazil (CAPES), finance code 001; Conselho Nacional de Desenvolvimento Científico e Tecnológico (CNPq, processes No. 428014/2018-6 and 434851/2018-3); and Fundação Araucária de Apoio ao Desenvolvimento Científico e Tecnológico do Estado do Paraná.

\section{Author Contributions}

Cristiane Guellis was responsible for the formal analysis, data curation, investigation, methodology, writing original draft; Ariane R. S. Rossin for the investigation; Guilherme G. Bessegato for the writing review and editing; Douglas C. Dragunski for the formal analysis and supervision; Reinaldo A. Bariccatti for the formal analysis and Cleber A. Lindino for the conceptualization, supervision, project administration, and writing review and editing.

\section{References}

1. Pakravan, M.; Heuzey, M. C.; Ajji, A.; Polymer 2011, 52, 4813.

2. de Vrieze, S.; Westbroek, P.; van Camp, T.; van Langenhove, L.; J. Mater. Sci. 2007, 42, 8029.
3. Bizarria, M. T. M.; D’ávila, M. A.; Mei, L. H. I.; Braz. J. Chem. Eng. 2014, 31, 57.

4. Homayoni, H.; Ravandi, S. A. H.; Valizadeh, M.; Carbohydr. Polym. 2009, 77, 656.

5. Corradini, E.; Curti, P. S.; da Silva Gonzalez, R.; Pereira, A. G. B.; Dragunski, D. C.; Martins, A. F.; Muniz, E. C. In Recent Advances in Complex Functional Materials: From Design to Application; Longo, E.; La Porta, F. A., eds.; Springer International Publishing: Cham, Switzerland, 2017, ch. 4.

6. Su, P.; Wang, C.; Yang, X.; Chen, X.; Gao, C.; Feng, X. X.; Chen, J. Y.; Ye, J.; Gou, Z.; Carbohydr. Polym. 2011, 84, 239.

7. Klossner, R. R.; Queen, H. A.; Coughlin, A. J.; Krause, W. E.; Biomacromolecules 2008, 9, 2947.

8. Mengistu Lemma, S.; Bossard, F.; Rinaudo, M.; Int. J. Mol. Sci. 2016, 17, 1790.

9. Chen, Q.; Xin, Z. X.; Saha, P.; Kim, J. K.; J. Polym. Eng. 2017, 37,461 .

10. Desai, K.; Kit, K.; Li, J.; Zivanovic, S.; Biomacromolecules 2008, 9, 1000.

11. Torres-Giner, S.; Ocio, M. J.; Lagaron, J. M.; Eng. Life Sci. 2008, 8, 303.

12. Sencadas, V.; Correia, D. M.; Areias, A.; Botelho, G.; Fonseca, A. M.; Neves, I. C.; Gomez Ribelles, J. L.; Lanceros Mendez, S.; Carbohydr. Polym. 2012, 87, 1295.

13. Martinová, L.; Lubasová, D.; Res. J. Text. Apparel 2008, 12, 72.

14. Li, L.; Hsieh, Y.-L.; Carbohydr. Res. 2006, 341, 374.

15. Pillai, C. K. S.; Paul, W.; Sharma, C. P.; Prog. Polym. Sci. 2009, 34,641 .

16. Song, Z.; Chiang, S. W.; Chu, X.; Du, H.; Li, J.; Gan, L.; Xu, C.; Yao, Y.; He, Y.; Li, B.; Kang, F.; J. Appl. Polym. Sci. 2018, $135,45787$.

17. Yan, X.; You, M. H.; Lou, T.; Yu, M.; Zhang, J. C.; Gong, M. G.; Lv, F. Y.; Huang, Y. Y.; Long, Y. Z.; Nanoscale Res. Lett. 2016, 11, 540.

18. Bailey, F. E.; Koleske, J. V.; Poly(Ethylene Oxide); Academic Press: New York, USA, 1976.

19. Schoolaert, E.; Steyaert, I.; Vancoillie, G.; Geltmeyer, J.; Lava, K.; Hoogenboom, R.; de Clerck, K.; J. Mater. Chem. B 2016, 4,4507 .

20. van der Schueren, L.; de Meyer, T.; Steyaert, I.; Ceylan, Ö.; Hemelsoet, K.; van Speybroeck, V.; de Clerck, K.; Carbohydr. Polym. 2013, 91, 284.

21. Wu, J. P.; Yin, F.; Anal. Lett. 2013, 46, 818.

22. Wu, J.; Yin, F.; J. Electroanal. Chem. 2013, 694, 1.

23. Horzum, N.; Mete, D.; Karakuş, E.; Üçüncü, M.; Emrullahoğlu, M.; Demir, M. M.; ChemistrySelect 2016, 1, 896.

24. dos Santos, D. M.; Bukzem, A. D. L.; Campana-Filho, S. P.; Carbohydr. Polym. 2016, 138, 317.

25. Pinto, L. C. M.; Quantikov Image Analyzer, v.18.5; IPEN/USP, Brazil, 1996. 
26. Nunes, C. A.; Chemoface, v.1.64; Universidade Federal de Lavras, Brazil, 2012.

27. Montgomery, D. C.; Introduction to Statistical Quality Control, $8^{\text {th }}$ ed.; John Wiley \& Sons: New York, USA, 2019.

28. TA-60WS Thermal Analysis Workstation, v.2.20; Labsolutions, Japan, 2020.

29. Kong, Y.; Hay, J. N.; Polymer 2002, 43, 3873.

30. Veleirinho, B.; Rei, M. F.; Lopes-Da-Silva, J. A.; J. Polym. Sci., Part B: Polym. Phys. 2008, 46, 460.

31. Min, B. M.; Lee, S. W.; Lim, J. N.; You, Y.; Lee, T. S.; Kang, P. H.; Park, W. H.; Polymer 2004, 45, 7137.

32. Zhang, C.; Yuan, X.; Wu, L.; Han, Y.; Sheng, J.; Eur. Polym. J. $\mathbf{2 0 0 5}, 41,423$.

33. Darbasizadeh, B.; Motasadizadeh, H.; Foroughi-Nia, B.; Farhadnejad, H.; J. Pharm. Biomed. Anal. 2018, 153, 63.

34. Zhang, J. F.; Yang, D. Z.; Xu, F.; Zhang, Z. P.; Yin, R. X.; Nie, J.; Macromolecules 2009, 42, 5278.

35. Markarian, S. A.; Shahinyan, G. A.; Spectrochim. Acta, Part A 2015, 151, 662.

36. Hubenko, K. O.; Yefimova, S. L.; Tkacheva, T. N.; Maksimchuk, P. O.; Sedyh, O. O.; Viagin, O. G.; Sorokin, A. V.; Malyukin, Y. V.; J. Fluoresc. 2018, 28, 943.

37. Karmakar, A.; Banerjee, S.; Singh, B.; Mandal, N. C.; J. Mol. Struct. 2019, 1177, 418.

38. Xu, Z.; Zheng, L.; Yin, Y.; Wang, J.; Wang, P.; Ren, L.; Eremin, S. A.; He, X.; Meng, M.; Xi, R.; Food Control 2015, 47, 472.

39. Pedro, M. F. C.; Kalck, A. S.; dos Santos, K. F.; Sousa, M. S.; Romio, K. B.; Souto, P. C. S.; Silva, J. R.; de Souza, N. C.; Photodiagn. Photodyn. Ther. 2018, 22, 158.

40. Łukarska, M.; Jankowska, A.; Gapiński, J.; Valable, S.; Anfray, C.; Ménard, B.; Mintova, S.; Kowalak, S.; Microporous Mesoporous Mater. 2018, 260, 70.

41. Szabelski, M.; Ilijev, D.; Sarkar, P.; Luchowski, R.; Gryczynski, Z.; Kapusta, P.; Erdmann, R.; Gryczynski, I.; Appl. Spectrosc. 2009, 63, 363.

42. Valeur, B.; Molecular Fluorescence: Principles and Applications; Wiley-VHC: Weinheim, Germany, 2002.

43. Sarkar, S.; Roy, S.; Sikdar, A.; Saha, R. N.; Panja, S. S.; Analyst 2013, 138, 7119.

44. Sorokin, A. V.; Gnap, B. A.; Bespalova, I. I.; Yefimova, S. L.; Malyukin, Y. V.; J. Lumin. 2016, 179, 171.

45. Lamola, A. A.; Turro, N. J. In Energy Transfer and Organic Photochemistry; Leermakers, P. A.; Weissberger, A., eds.; Interscience Publishers: New York, USA, 1969.

46. Kohsari, I.; Shariatinia, Z.; Pourmortazavi, S. M.; Int. J. Biol. Macromol. 2016, 91, 778.

47. Queiroz, M. F.; Melo, K.; Sabry, D.; Sassaki, G.; Rocha, H.; Mar. Drugs 2014, 13, 141.
48. Deng, L.; Taxipalati, M.; Zhang, A.; Que, F.; Wei, H.; Feng, F.; Zhang, H.; J. Agric. Food Chem. 2018, 66, 6219.

49. de Farias, B. S.; Vidal, É. M.; Ribeiro, N. T.; da Silveira, N.; Vaz, B. S.; Kuntzler, S. G.; de Morais, M. G.; Cadaval, T. R. S. A.; Pinto, L. A. A.; J. Mol. Liq. 2018, 268, 365.

50. Branca, C.; D’Angelo, G.; Crupi, C.; Khouzami, K.; Rifici, S.; Ruello, G.; Wanderlingh, U.; Polymer 2016, 99, 614.

51. Wang, P.; Wang, L.; Dong, S.; Zhang, G.; Shi, X.; Xiang, C.; Li, L.; New J. Chem. 2018, 42, 17740.

52. Aluigi, A.; Varesano, A.; Montarsolo, A.; Vineis, C.; Ferrero, F.; Mazzuchetti, G.; Tonin, C.; J. Appl. Polym. Sci. 2007, 104, 863.

53. Pereira, V. A.; de Arruda, I. N. Q.; Stefani, R.; Food Hydrocolloids 2015, 43, 180.

54. Bonardd, S.; Schmidt, M.; Saavedra-Torres, M.; Leiva, A.; Radic, D.; Saldías, C.; Carbohydr. Polym. 2016, 144, 315.

55. Amico, V.; Meille, S. V.; Corradi, E.; Messina, M. T.; Resnati, G.; J. Am. Chem. Soc. 1998, 120, 8261.

56. Dumas, J.-M.; Gomel, L.; Guerin, M. In Halides, Pseudo Halides and Azides (1983): Part 2, vol. 2; Patai, S.; Rappoport, Z., eds.; John Wiley \& Sons: New York, USA, 1983, p. 985.

57. Kuntzler, S. G.; Costa, J. A. V.; de Morais, M. G.; Int. J. Biol. Macromol. 2018, 117, 800.

58. Duan, B.; Dong, C.; Yuan, X.; Yao, K.; J. Biomater. Sci., Polym. Ed. 2004, 15, 797.

59. Wei, H.; Zhang, F.; Zhang, D.; Liu, Y.; Leng, J.; J. Appl. Polym. Sci. 2015, 132, 42532.

60. Bostan, M. S.; Mutlu, E. C.; Kazak, H.; Sinan Keskin, S.; Oner, E. T.; Eroglu, M. S.; Carbohydr. Polym. 2014, 102, 993.

61. Garapati, C.; Clarke, B.; Zadora, S.; Burney, C.; Cameron, B. D.; Fournier, R.; Baugh, R. F.; Boddu, S. H. S.; Photodiagn. Photodyn. Ther. 2015, 12, 9.

62. Yassunaka, N. N.; de Freitas, C. F.; Rabello, B. R.; Santos, P. R.; Caetano, W.; Hioka, N.; Nakamura, T. U.; de Abreu Filho, B. A.; Mikcha, J. M. G.; Curr. Microbiol. 2015, 71, 243.

63. Lozano-Torres, B.; Pascual, L.; Bernardos, A.; Marcos, M. D.; Jeppesen, J. O.; Salinas, Y.; Martínez-Máñez, R.; Sancenón, F.; Chem. Commun. 2017, 53, 3559.

64. Houtman, C. J.; Kitin, P.; Houtman, J. C. D.; Hammel, K. E.; Hunt, C. G.; PLoS One 2016, 11, e0159715.

65. Powless, A. J.; Conley, R. J.; Freeman, K. A.; Muldoon, T. J.; J. Biomed. Opt. 2017, 22, 035001.

66. Amado, A. M.; Ramos, A. P.; Silva, E. R.; Borissevitch, I. E.; J. Lumin. 2016, 178, 288.

Submitted: September 24, 2020 Published online: January 12, 2021 